PREPARED FOR THE U.S. DEPARTMENT OF ENERGY, UNDER CONTRACT DE-AC02-76CH03073

PPPL-3500

PPPL-3500

UC-70

Numerical Study of Global Stability of Oblate

Field-Reversed Configurations

by

E.V. Belova, S.C. Jardin, H. Ji, M. Yamada, and R. Kulsrud

October 2000
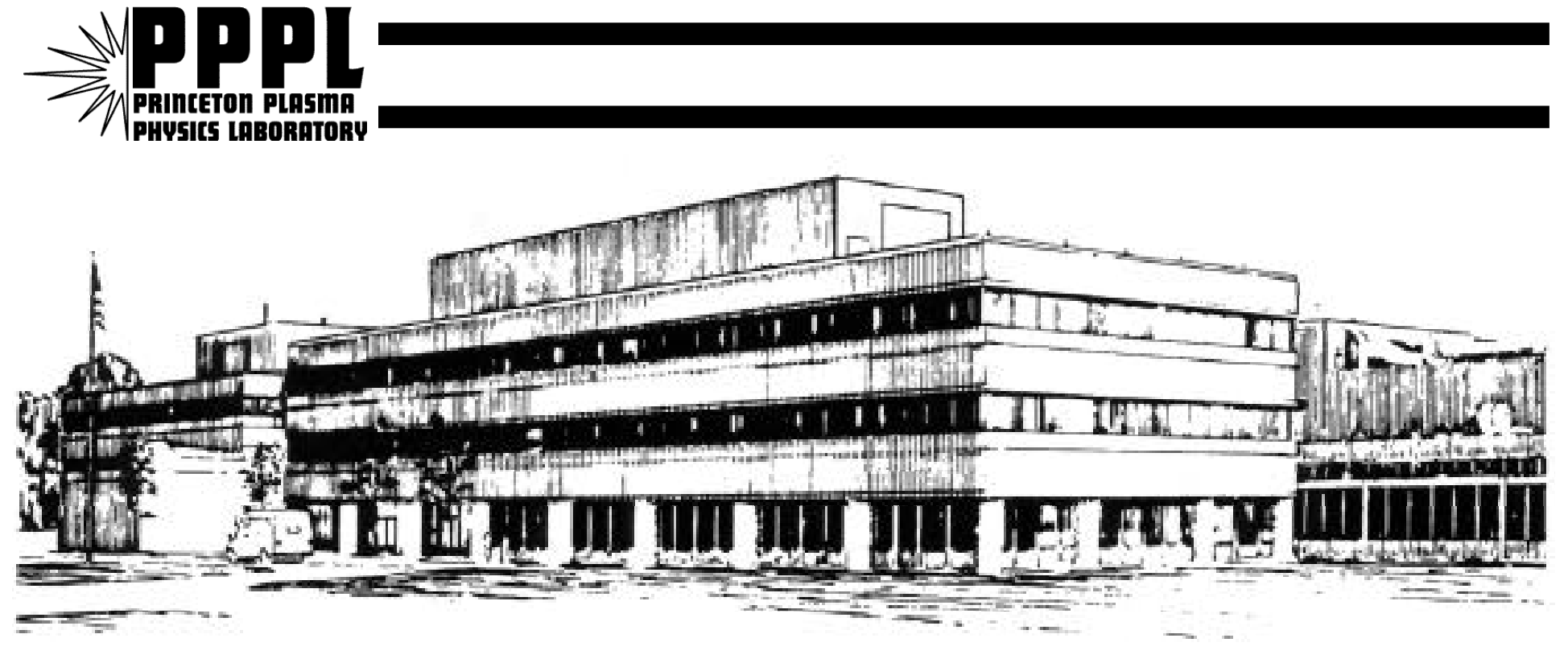

PRINCETON PLASMA PHYSICS LABORATORY PRINCETON UNIVERSITY, PRINCETON, NEW JERSEY 


\section{PPPL Reports Disclaimer}

This report was prepared as an account of work sponsored by an agency of the United States Government. Neither the United States Government nor any agency thereof, nor any of their employees, makes any warranty, express or implied, or assumes any legal liability or responsibility for the accuracy, completeness, or usefulness of any information, apparatus, product, or process disclosed, or represents that its use would not infringe privately owned rights. Reference herein to any specific commercial product, process, or service by trade name, trademark, manufacturer, or otherwise, does not necessarily constitute or imply its endorsement, recommendation, or favoring by the United States Government or any agency thereof. The views and opinions of authors expressed herein do not necessarily state or reflect those of the United States Government or any agency thereof.

\section{Availability}

This report is posted on the U.S. Department of Energy's Princeton Plasma Physics Laboratory Publications and Reports web site in Calendar Year 2000. The home page for PPPL Reports and Publications is: http://www.pppl.gov/pub_report/

DOE and DOE Contractors can obtain copies of this report from:

U.S. Department of Energy

Office of Scientific and Technical Information

DOE Technical Information Services (DTIS)

P.O. Box 62

Oak Ridge, TN 37831

Telephone: (865) 576-8401

Fax: (865) 576-5728

Email: reports@adonis.osti.gov

This report is available to the general public from:

National Technical Information Service

U.S. Department of Commerce

5285 Port Royal Road

Springfield, VA 22161

Telephone: $1-800-553-6847$ or

(703) $605-6000$

Fax: (703) 321-8547

Internet: http://www.ntis.gov/ordering.htm 


\title{
Numerical Study of Global Stability of Oblate Field-Reversed Configurations
}

\author{
E. V. Belova, S. C. Jardin, H. Ji, M. Yamada, R. Kulsrud \\ Princeton Plasma Physics Laboratory, Princeton, NJ 08543, USA
}

\begin{abstract}
Global stability of the oblate (small elongation, $E<1$ ) Field-Reversed Configuration (FRC) has been investigated numerically using both three-dimensional magnetohydrodynamic (MHD) and hybrid (fluid electrons and kinetic ions) simulations. For every non-zero value of the toroidal mode number $n$, there are three MHD modes that must be stabilized. For $n=1$, these are the interchange, the tilt and the radial shift; while for $n>1$ these are the interchange and two co-interchange modes with different polarization. It is shown that the $n=1$ tilt mode becomes an external mode when $E<1$, and it can be effectively stabilized by close-fitting conducting shells, even in the small Larmor radii (MHD) regime. The tilt mode stability improves with increasing oblateness, however at sufficiently small elongations the radial shift mode becomes more unstable than the tilt mode. The interchange mode stability is strongly profile dependent, and all $n \geq 1$ interchange modes can be stabilized for a class of pressure profile with separatrix beta larger than 0.035. Our results show that all three $n=1$ modes can be stabilized in the MHD regime, but the stabilization of the $n>1$ co-interchange modes still remains an open question.
\end{abstract}

PACS numbers: 52.35.Py, 52.55.Hc, 52.65.Rr, 52.65.Kj 


\section{INTRODUCTION}

The Field-Reversed Configuration (FRC) is a compact toroid with negligible toroidal field, in which plasma is confined by a poloidal magnetic field associated with toroidal diamagnetic current carried by the plasma. The unique fusion reactor potential of the FRC (compact and simple geometry, translation properties and high plasma beta) has motivated extensive experimental and theoretical research ${ }^{1-11}$. Most of the work done so far has been concentrated on elongated (prolate) configurations with elongation $E \gtrsim 2$, where elongation is defined as the ratio of the separatrix half-length to its radius, $E=Z_{s} / R_{s}$. One of the important issues considered is the FRC stability with respect to low $n$ magnetohydrodynamic (MHD) modes, where $n$ is toroidal mode number.

There is a clear discrepancy between the predictions of standard MHD theory that many modes should be unstable on the MHD time scale, and the observed macroscopic resilience of FRCs in experiments, where prolate FRCs have been produced successfully by several formation techniques with lifetimes on the order of hundreds of Alfvén times ${ }^{1}$. Since most FRC experiments have been performed in the kinetic regime, with small values of the parameter $\bar{s}(\bar{s} \sim 1-4)$, this discrepancy is commonly attributed to kinetic effects, missing from the standard MHD model. Here the kinetic parameter $\bar{s}$ measures the number of ion Larmor gyroradii in the configuration, and is defined by

$$
\bar{s}=\int_{R_{0}}^{R_{s}} \frac{r d r}{R_{s} \rho_{i}},
$$

where $R_{0}$ and $R_{s}$ are the magnetic null and the separatrix radius at the midplane, and $\rho_{i}$ is the local thermal ion Larmor radius. The parameter $\bar{s}$ indicates the importance of finite Larmor radius (FLR) effects, which are strong for $\bar{s} \sim 1$, while the $\bar{s} \gg 1$ limit corresponds to the MHD regime. Numerous calculations including kinetic effects have been performed in order to explain the observed stability of prolate $\mathrm{FRCs}^{3-7}$. A theoretical picture is now emerging of how kinetic effects can stabilize the low $n$ modes for small enough values of $\bar{s}$, in particular the $n=1$ internal tilt mode $^{8}$.

While the mechanism providing low $\bar{s}$ stability to prolate FRCs is of considerable scientific interest, the fusion reactor potential of the FRC is enhanced significantly if this stability 
is extended to the large $\bar{s}(\bar{s} \sim 20-40)$ MHD-like regime. While the gross stability of prolate FRCs at large $\bar{s}$ cannot be completely ruled out, it has yet to be demonstrated either experimentally or theoretically. Thus, we are motivated to study an extended class of FRC configurations for stability at large $\bar{s}$.

Oblate FRCs $(E<1)$ are known to have different stability properties than prolate $\mathrm{FRCs}^{9-12}$. In particular, the $n=1$ internal tilt mode in the prolate FRC becomes an external mode for oblate configurations, and thus can potentially be stabilized by closefitting conducting shells, even in the large $\bar{s}$ MHD regimes. In spheromak experiments, for example, it has been demonstrated that the tilt mode can be suppressed by figure- 8 coils ${ }^{13}$ when the plasma shape is oblate ${ }^{14}$. An experimental study of oblate FRC stability has been performed recently in the merging experiments in Japan ${ }^{15}$. In these experiments, a slow-formation of the FRC has been developed using two merging spheromaks with opposite magnetic helicities. It has been reported that the stability of the $n=1$ tilt mode has been greatly improved by a combination of the oblate shape, low $\bar{s}$, and insertion of a central conductor and transformer.

These considerations motivated the present numerical work, in which the possible means of stabilization of the oblate FRCs are investigated using three-dimensional (3D) nonlinear MHD and hybrid (fluid electrons and kinetic particle ions) simulations. For every non-zero value of the toroidal mode number $n$, there are three MHD modes that must be stabilized. For $n=1$, these are the interchange (or sausage), the tilt and the radial shift; while for $n>1$ these are the interchange and two co-interchange modes with different polarization. The effects of the separatrix shaping (elongation), conducting shells, profile effects, and kinetic effects on the stability of these modes are all considered. The linear stability of the $n=1$ modes in oblate FRCs is discussed in detail in the next Section. Simulation results concerning the nonlinear evolution and stability of the $n>1$ modes are presented in the Section III, and results of the hybrid simulations are described in Section IV. Conclusions and discussion are given in Section $\mathrm{V}$.

\section{LINEAR $n=1$ MODES}

There are three types of $n=1$ modes which can be unstable in oblate FRC: the tilt 
mode, which has an odd symmetry of $v_{r}$ relative to midplane; and two even modes - the radial shift mode and an interchange (sausage) mode.
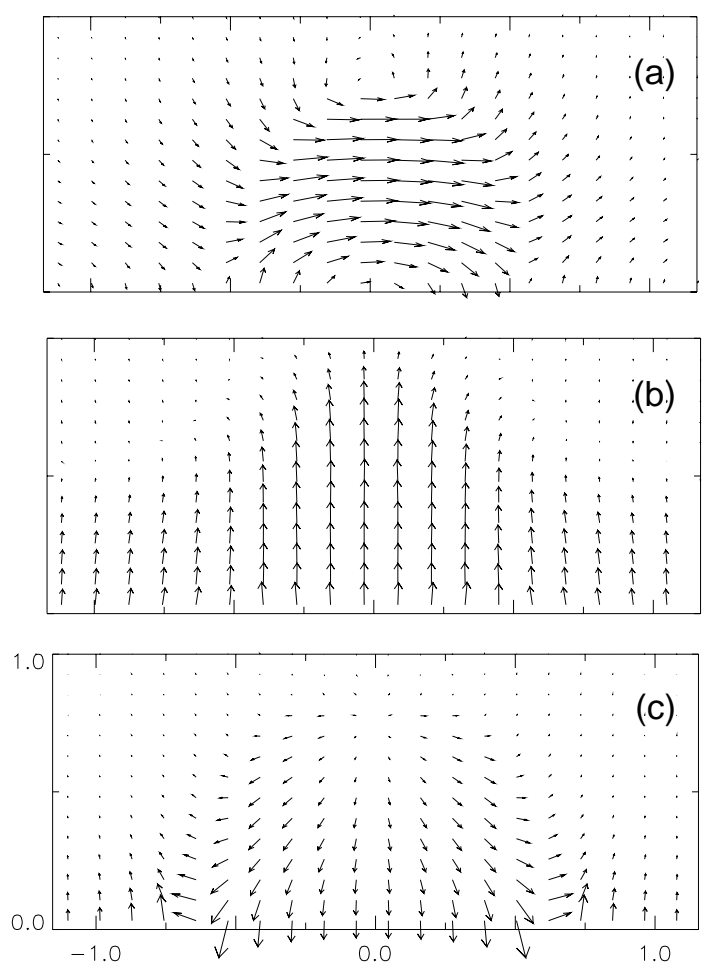

Fig.1 Poloidal mode structure of the $n=1$ (a) tilt, (b) radial shift, and (c) interchange modes, obtained in the different linear MHD simulations with $E=0.6-0.7$. Shown are vector plots of the poloidal velocity in $r-z$ plane.

Typical poloidal structures of these modes are shown in Fig. 1, where vector plots of the poloidal component of the fluid velocity obtained in MHD simulations with different pressure profiles and initial perturbations and with $E \sim 0.6-0.7$, are shown. In this Section the possibility of stabilization of these $n=1$ modes in the MHD regime is discussed.

\section{A. Numerical model}

The MHD simulations were performed using the MHD version of our FRC stability code described earlier ${ }^{7,11}$. In the MHD version of the code, the nonlinear resistive one-fluid MHD equations are advanced using a second-order, explicit, time-stepping scheme with fourth-order accurate spatial derivatives. A cylindrical grid is employed and finite difference 
approximations are used in all three directions $(r, \phi, z)$. A fine resolution of $(100 \times 32 \times 150)$ grid points has typically been used in the MHD runs. Periodic boundary conditions are applied in both the toroidal and axial directions, and geometric boundary conditions are implemented at $r=0$. The cylinder length is taken to be large enough, so that periodic boundary conditions in the axial direction have no effect on the stability. At the radial wall, $r=R_{c}$, (or at the conformal shell location, if present) perfectly conducting boundary conditions for the velocity and non-equilibrium component of the field are used, $(\delta \mathbf{V}=0$, $\delta \mathbf{A}=0)$. A two-dimensional (2D) self-consistent equilibrium is found by solving the GradShafranov equation for the field and plasma pressure including equilibrium flows, and this is used as an initial condition for 3D stability calculations. The plasma temperature is taken as constant throughout the plasma so that the density varies as $\rho \sim p$. The vacuum region is treated as a low density plasma region, and a small cutoff density (typically 0.05 to 0.1 fraction of the peak density) is applied to avoid the severe time step restrictions that the Courant condition would impose. A nonuniform resistivity profile with resistivity inversely proportional to the plasma density is used, so that the "vacuum" is modeled as a highly resistive, low density plasma. The linearized form of the MHD equations has been included in the code as an option, and this has been used for the stability studies of marginally unstable modes.

\section{B. Tilting and shifting modes}

The $n=1$ tilt instability is thought to be the most dangerous MHD instability in prolate FRCs because it is a global internal mode which cannot easily be stabilized by kinetic effects or by external means. However, it was shown both analytically and in numerical spectral stability calculations that the internal tilt is stable when $E \leq 1$. Nevertheless, two other $n=1$ free boundary modes, the external tilt mode and the radial shift mode can be unstable when the separatrix shape is oblate. It was found by Rosenbluth and Bussac ${ }^{12}$ that a closefitting conducting shell can stabilize these modes in oblate spheromak configurations. In this work we have performed MHD simulations to study the stabilization of the external tilt and shift modes in oblate FRCs with $E \leq 1$.

Our simulations show that the growth rate of the tilt instability is not very sensitive 
to the equilibrium pressure profiles, and that it is approximately equal to $\gamma_{0}=V_{A} / Z_{s}$ for elongations $E \sim 1$. Here $V_{A}$ is the Alfvén velocity defined by $V_{A}=B_{0} / \sqrt{4 \pi \rho_{0}}, B_{0}$ is the magnetic field amplitude at $r=0$ at the midplane, $\rho_{0}$ is the plasma density at the magnetic null, and $Z_{s}$ is the separatrix half-length at $r=0$. The tilt and the radial shift mode calculations have been performed for a plasma pressure profile

$$
p(x)=0.5 p_{0}[\tanh (a x+b)+1], \quad x=\psi / \psi_{0} .
$$

Here $\psi_{0}$ is the value of the flux at the magnetic null point $\left(\psi_{0}<0\right)$, and $a=1.5, b=-3.3$ are the pressure profile parameters. Note that $x>0$ inside the separatrix and $x<0$ outside of it. For this equilibrium, the separatrix beta is finite, but small, $p(0) / p(1)=0.05$. The separatrix radius at the midplane is $x_{s}=R_{s} / R_{c} \approx 0.7$.

It has been suggested earlier that the external tilt instability in the FRC is suppressed when the separatrix elongation is small enough ${ }^{10}$. The stabilization is provided by the strong shaping vacuum fields, which are needed to form the strongly oblate FRC (with $E \lesssim 0.5)$. The strong curvature of these mirror-like fields provides a restoring force to the tilt-like perturbation. Our simulations confirm this stabilization. However we find that when $E \lesssim 0.5$ a radial shift mode becomes unstable with a growth rate that increases as $E$ decreases.

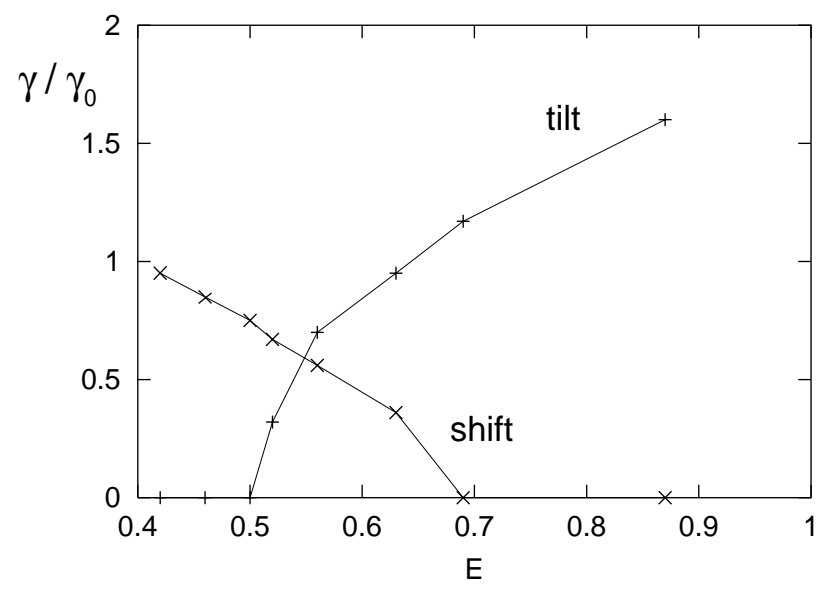

Fig.2 Growth rates of the tilt and the radial shift instabilities versus elongation. 
Fig. 2 shows the tilt and the radial shift mode growth rates calculated in the linearized MHD simulations for different separatrix elongations using the equilibrium pressure profile given by Eq. (1). The elongation was changed from $E=0.87$ to $E=0.42$ by varying the mirror ratio of the external field from $r_{m}=1.0$ to $r_{m}=2.4$, while keeping the amplitude of the reversed field on the axis (at the midplane) and the separatrix radius the same. (The mirror ratio $r_{m}$ is defined as the ratio of the magnitude of vacuum magnetic field on the axis near the end of the cylinder to that at the midplane, $r_{m}=B\left(r=0, z=Z_{c}\right) / B(r=0, z=0)$.) As the elongation decreases, the growth rate of the tilt mode becomes smaller, while the growth rate of the radial shift mode increases. As the elongation decreases below $E=0.55$, the shift mode becomes more unstable than the tilt mode. Note that this is very similar to the behavior of tilt and shift instabilities in a spheromak ${ }^{16,17}$, where the mode crossing occurs approximately at $E=0.6$. As can be seen from Fig. 2, at the mode crossing point the growth rate is reduced only by a factor of three from that of the tilt mode growth rate for the equilibrium with $E \sim 1$. However, the poloidal structure of the tilt mode changes significantly as the elongation changes from $E=0.7$ to $E=0.5$. At $E=0.69$ the tilt mode structure is shown in Fig. 1(a). At smaller $E$, the mode is localized near $r=0$, and becomes nearly singular at the $\mathrm{X}$ points.

\section{Wall stabilization}

The possibility of stabilizing the tilt mode for elongations in the range $1>E>0.5$ by placing a conducting shell close to the separatrix has been investigated in MHD simulations. In these simulations the pressure profile was taken to be

$$
p(\psi)=p_{0} \begin{cases}0.5 x^{2}[\tanh (a x+b)-\tanh (b)] & x>0 \\ 0 & x \leq 0\end{cases}
$$

For this profile, the plasma pressure and toroidal current are both equal to zero at the separatrix and in the external region. Simulations have been carried out for $a=3.0, b=$ -0.5 , and the external field mirror ratio $r_{m}=1.5$ corresponding to elongation $E=0.64$. Both uniform resistivity and resistivity inversely proportional to the equilibrium plasma density (normalized to the null-point) have been used. 

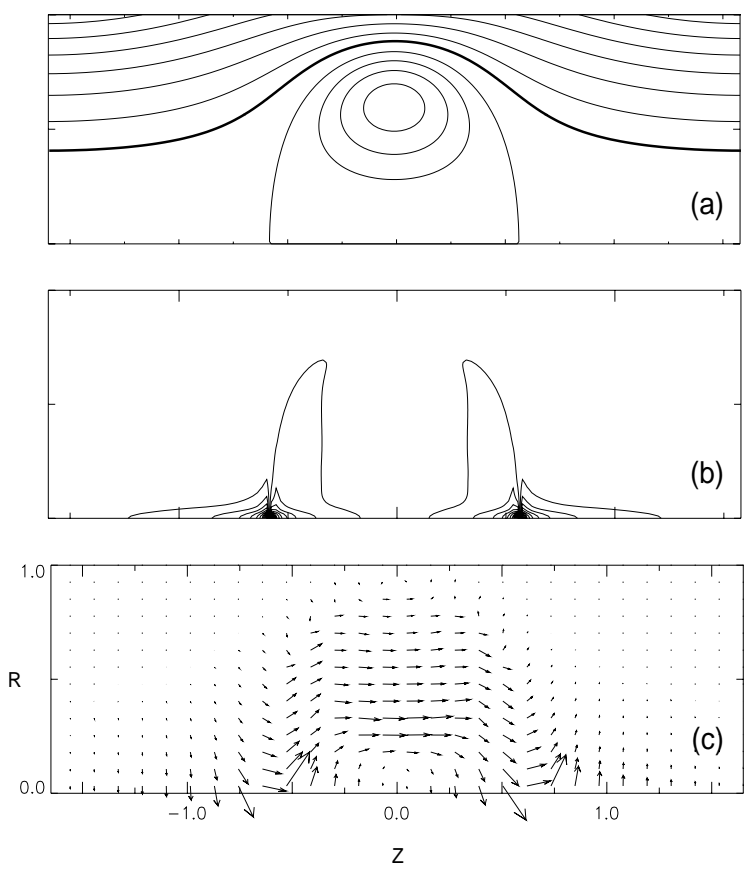

Fig.3 (a) Equilibrium flux contours from the simulations with $E=0.64$; the position of the conducting wall $(d=0.06)$ is indicated by a thick line. Tilt mode structure obtained in the simulation with $d=0.07$ : (b) contour plot of toroidal velocity component $V_{\phi}$, and (c) vector plot of poloidal velocity.

The equilibrium poloidal flux contours for these calculations are shown in Fig. 3(a), where the thick line indicates the position of the conformal conducting shell, chosen to follow an external flux surface. The 2D equilibria for this set of simulations is calculated in a full cylinder as before, but perfectly conducting boundary conditions are applied at the shell in the 3D simulations.

The calculated growth rate of the tilt mode is shown in Fig. 4 as a function of a separation $d$ between the separatrix and conducting wall for uniform resistivity (solid line) and nonuniform resistivity (dashed line). Here the parameter $d=\left(R_{w}-R_{s}\right) / R_{s}$ is defined as the distance from the separatrix to the conformal conducting wall normalized to the separatrix radius, as calculated at the midplane. The value of $d=0.2$ corresponds to the case with no conducting wall inside the cylinder $\left(R_{w} \geq R_{c}\right)$. As can be seen from Fig. 4 , the growth rate is reduced when the conducting wall is moved in closer to the separatrix, and 
complete stabilization occurs at the separation $d \leq 0.06$.

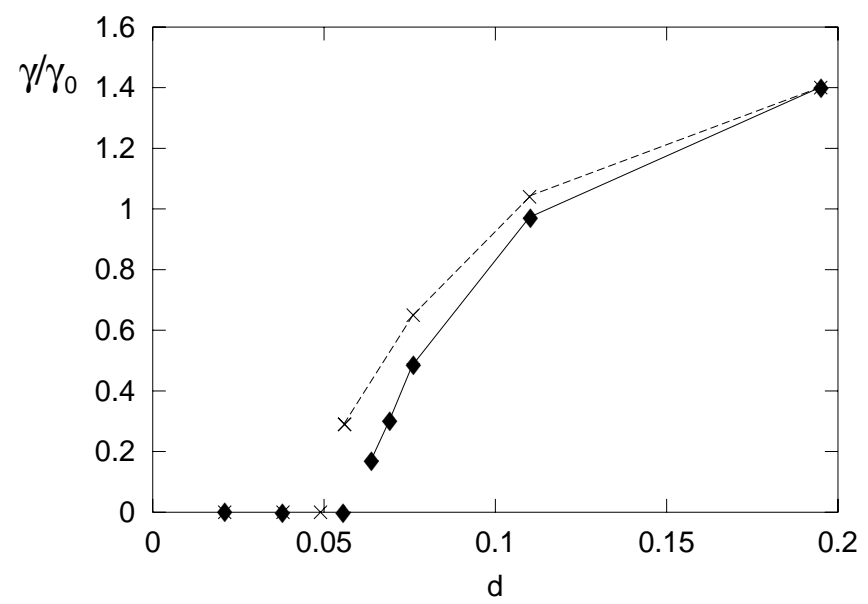

Fig.4 Tilting instability growth rate as a function of the separation between the conducting wall and the separatrix, $d=\left(R_{w}-R_{s}\right) / R_{s}$, for uniform resistivity (solid line), and resistivity profile inversely proportional to the equilibrium plasma density (dashed line).

Another way to specify the conducting wall position is by the value of the flux on the corresponding flux surface. The data points indicated on Fig. 4 correspond to the flux values ranging from $\psi=5 \%$ to $\psi=100 \%$ of the maximum flux at the cylinder wall $\left(\psi_{m}\right.$ at $z=Z_{c}$ and $\left.r=R_{c}\right)$, where the marginal separation $(d=0.06)$ occurs at $\psi=15 \%$ of $\psi_{m}$ (for uniform resistivity). The position of the conformal conducting wall corresponding to marginal stability with $d=0.06$ is shown on Fig. 3(a). The poloidal structure of the tilt mode from the calculations close to the marginal stability $(d=0.07)$ is displayed on Fig. 3(b,c), where the contour plot of the toroidal velocity component and the vector plot of poloidal velocity are shown.

The separation at which the tilt mode becomes stabilized depends somewhat on the resistivity profile, and as the resistivity of the plasma between the magnetic separatrix and the conducting shell is increased, the shell has to be moved closer to the separatrix for stability. The results shown in Fig. 3 and Fig. 4 (solid line) have been obtained in the simulations for a uniform resistivity, which corresponds to Lundquist number $S=R_{c} V_{A} / \eta=2.5 \cdot 10^{3}$. In the same set of simulations with resistivity profile taken to be inversely proportional to the 
equilibrium density, tilt stability has been obtained for the conducting wall position corresponding to $d \leq 0.05\left(\psi=0.12 \psi_{m}\right)$. In these calculations, the cutoff density was $n_{c}=0.1 n_{0}$, and the resistivity of the plasma outside the separatrix was about an order of magnitude larger than that in the simulations with a uniform resistivity profile. From Fig. 4 it is seen that, when the separation between the conducting wall and the separatrix is large, the tilt growth rate is independent of the resistivity, as expected for an ideal instability. However, growth rates at small separations do depend on the plasma resistivity on the open field lines, indicating that the instability persists as a resistive one when it is stabilized in ideal $\mathrm{MHD}^{18}$.

The conducting wall stabilization also depends on the current profile close to the separatrix. Configurations with toroidal current reducing smoothly to zero near the separatrix have been found to be more stable than configurations with significant current at the separatrix. The increased stability of the equilibria with zero or small current near the separatrix can be understood by considering the forces driving the instability. The tilt mode structure from the calculations performed near marginal stability is shown in Fig. 3(b,c). The singular behavior near the $\mathrm{X}$ points seen in Fig. 3(b,c) can be explained by a magnetic pressure imbalance across the perturbed X points, which is produced by the tilt motion and drives the instability ${ }^{12}$. Since the destabilizing force is localized near the separatrix, equilibria with a flux hole or with the current reducing smoothly to zero near the separatrix are more stable. This behavior is similar to the results obtained regarding the tilt mode in spheromaks, where the stabilizing influence of the flux hole and the current smoothing in the neighborhood of the separatrix were shown ${ }^{16,17}$.

Note that the X-point localized structure of the tilt mode seen on Fig.3(b,c), can be an important factor in the recently proposed tilt stabilization mechanism ${ }^{19}$. This mechanism takes into account the non-fluid behavior of the electrons trapped in the large curvature regions close to X points and executing fast toroidal drift motion. Since this drift frequency is large compared to the mode frequency, the electrons do not respond to the tilt perturbation in an MHD way. The ion motion becomes restricted as well due to the quasi-neutrality constraint, limiting the class of possible MHD perturbations. The self-consistent examination of this effect will require a kinetic description for the electrons which is not included in our 
present model.

In addition to the conducting wall stabilization described above, the effect of a central conductor on the tilt mode stability has also been investigated in MHD simulations with $E=0.63$. The central stack was assumed to be a perfectly conducting cylinder of radius $r_{c}$. Line tying effects were included (that is, the equilibrium magnetic field lines were allowed to cross the surface of the conductor). Considerable reduction in the instability growth rate was observed under these conditions. In particular, for the values of $r_{c} / R_{c}=0.0,0.15$, and 0.20 the tilt mode growth rate was calculated to be $\gamma / \gamma_{0}=0.96,0.68$, and 0.55 . Thus the growth rate is reduced almost by a factor of two when the radius of the central conductor is $20 \%$ of the external wall radius. These calculations are consistent with the results of the oblate FRC experiments ${ }^{15}$, where the insertion of a central conductor has been found to improve the tilt mode stability.

\section{Interchange mode stability}

Our MHD simulations have shown that unlike the tilt and radial shift modes, the $n=1$ interchange (sausage) mode cannot easily be stabilized by a close fitting conducting shell, even when the shell is placed at the separatrix. However, the stability to interchange modes is sensitive to the precise distribution of plasma pressure ${ }^{20}$. We have performed MHD simulations for several FRC equilibria with different pressure profiles and different values of the separatrix beta, $\beta_{s}$, in order to investigate their effect on the $n=1$ interchange mode.

In axisymmetric field reversed equilibria, there exists a necessary condition for stability against pure interchange modes ${ }^{21}$

$$
\left(V^{\prime \prime} / V^{\prime}+p^{\prime} / \Gamma p\right)>0
$$

where $V$ is the volume within a $\psi=$ const surface, $V^{\prime}=2 \pi \oint d l / B$ and primes denote derivatives with respect to poloidal flux, $\psi$, and $\Gamma=5 / 3$ is the ratio of specific heats. The derivation of this condition assumes pure interchange displacements for which $n \gg 1$ and the normal displacement $X \sim \xi \cdot \nabla \psi$ ( $\xi$ is the displacement vector) is constant along the magnetic field line. Thus this condition does not apply to the stability of modes with ballooning structure or to tilt and radial shift modes. The condition (3) was derived in the limit $n \gg 1$, and it can be shown that for these assumptions higher $n$ interchange modes are 
more unstable than the $n=1$ interchange mode.

The first term in (3) is positive and logarithmically diverges at $\psi=0$, therefore a finite value of $p^{\prime} / p(p \neq 0)$ at the separatrix is sufficient for the pure interchange stability of the flux surfaces near the separatrix. Since the value of the negative term $p^{\prime} / \Gamma p$ in (3) can be reduced by increasing the plasma pressure, $p$, configurations with larger pressure at the separatrix should be, in general, more stable ${ }^{20}$.

In order to investigate the dependence of the $n=1$ interchange mode growth rate on the value of $p_{s}=p(0) / p(1)$ (the ratio of the separatrix pressure to the pressure at the magnetic null point), we have performed linearized MHD simulations using the equilibrium pressure profile given by Eq. (2) and with $E=0.64$. This is the same profile used in the conducting wall stabilization study of the tilt mode, described above. The linearized version of our MHD code has been used in these simulations in order to avoid the growth of $n>1$ modes. The initial perturbation $(n=1)$ of even symmetry (in terms of $v_{r}$ ) relative to the midplane was applied at $t=0$. Thus the tilt mode (odd symmetry) did not grow, and the growth rate of the radial shift mode for this equilibrium was much smaller than that of the interchange mode. The value of $p_{s}$ in the simulations was changed by adding a constant term to the $p(\psi)$ profile, which corresponds to adding a uniform background pressure, and thus does not alter the computed equilibria. Our simulations show that as $p(0) / p(1)$ is increased, the growth rate of the $n=1$ interchange mode decreases, and the mode becomes more localized near the magnetic null.

The results of these simulations are shown in Figs. 5 and 6 , where the dependence $\gamma\left(p_{s}\right)$ is shown, as well as the change in the linear mode structure with $p_{s}$. The growth rate is reduced by an order of magnitude, as the separatrix pressure increases from zero to $0.3 p_{\max }$. Figure 6 shows the vector plots of the poloidal velocity in the $r-z$ plane from the simulations with $p_{s}=0.125$ and $p_{s}=0.29$. For comparison, the interchange mode structure for the same equilibrium and zero separatrix pressure, $p_{s}=0.0$, is shown in Fig. 1(c). From Fig. 6 it is seen that as $p_{s}$ is increased, the interchange mode eigenfunction first vanishes at the flux surfaces near the separatrix. When the plasma pressure at the separatrix is large enough, the eigenfunction vanishes everywhere and the configuration becomes stable with respect to 
all interchange modes.

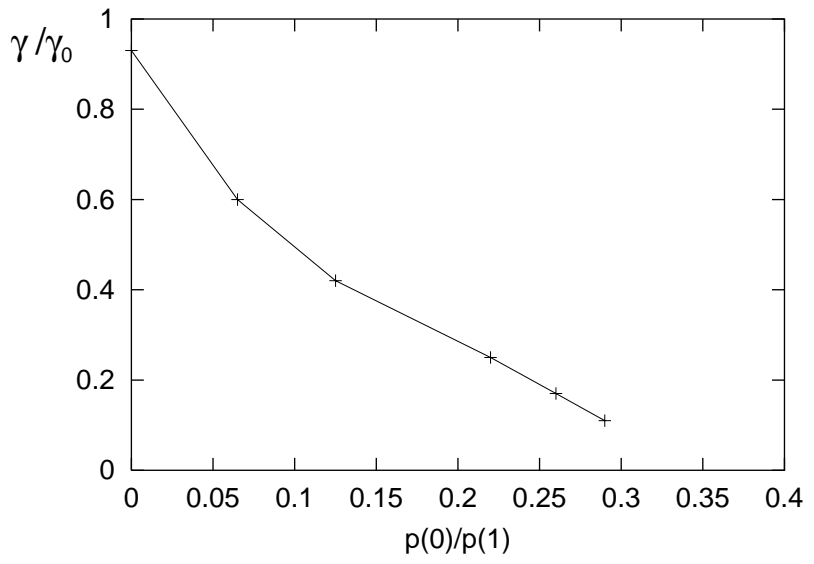

Fig. 5 Growth rate of the $n=1$ interchange (sausage) mode as a function of the separatrix pressure $p_{s}=p(0) / p(1)$ for the pressure profile given by Eq. (2).
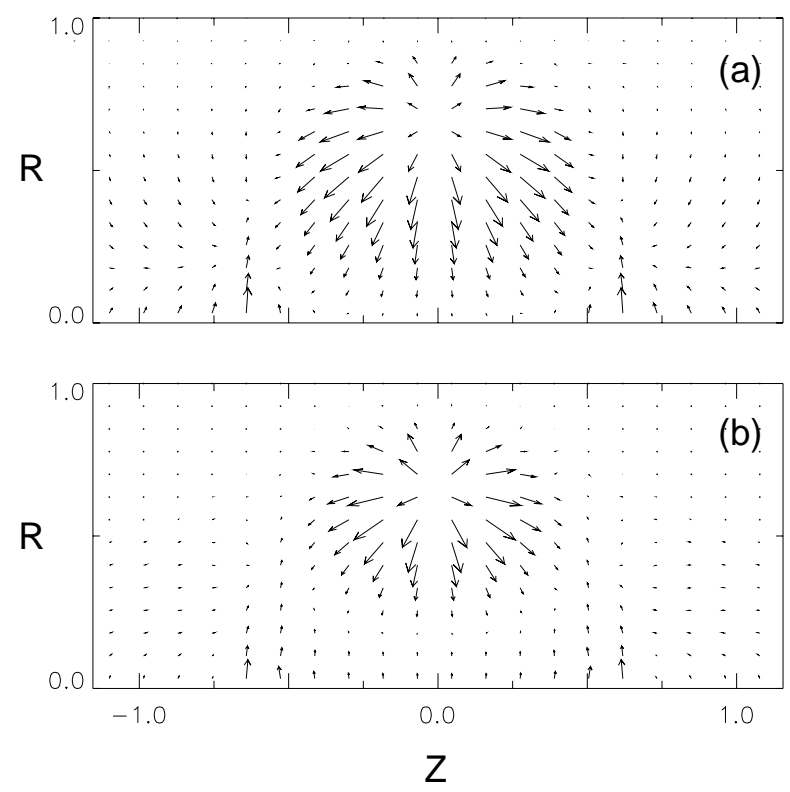

Fig.6 The $n=1$ interchange mode structure for (a) $p_{s}=0.125$, and (b) $p_{s}=0.29$ for the same pressure profile as calculations in Fig. 5.

For the pressure profile given by Eq. (2), the $n=1$ stability occurs at $p(0) / p(1) \gtrsim 0.35$, as seen in Fig. 5. Note that $p_{s}>0$ implies a finite value of plasma density on the open field lines. While there may be experimental reasons to expect this, for example due to finite ion 
Larmor radius or an externally applied mirror field, it is still desirable to find an interchange stable equilibrium with a small value of $p(0)$, especially if a close-fitting conducting wall is to be used for tilt mode stabilization.

Previous calculations ${ }^{20}$ show that the value of $p_{s}$ at which the interchange mode becomes marginally stable depends strongly on the pressure profile, and can vary from $p_{s}<0.1$ to $p_{s} \sim 1$. In order to study how this value depends on the profile parameters, we have evaluated the interchange stability criterion, Eq. (3), on the internal flux surfaces using TSC (Tokamak Simulation Code) $\operatorname{code}^{22}$.
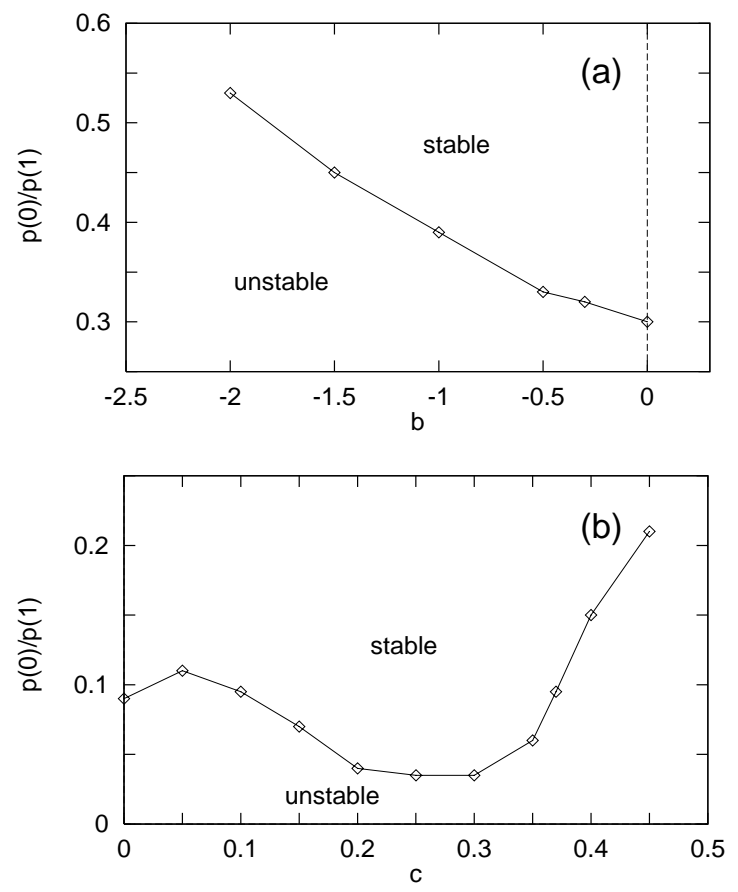

Fig.7 Interchange stability diagram for two different pressure profiles given by Eq. (2) (a); and by Eq. (4) - (b).

The results of these calculations are summarized in Fig. 7, where the marginal stability curves for two different pressure profiles are shown. For the same pressure profile as used in the above MHD simulations, Eq. (2), the stability diagram is shown in Fig. 7a, where the value of $p_{s}$ is plotted versus the profile parameter $b$ (larger value of $|b|$ corresponds to a more peaked pressure profile and smaller elongation). Figure 7a indicates that for the equilibrium with pressure profile given by Eq. (2) a large pressure on the separatrix, $p(0) \gtrsim 0.3 p_{\max }$, 
is required to stabilize the interchange modes. Note that for $b=-0.5$, there is a good agreement between the initial value MHD simulations (Fig. 5) and the interchange stability criteria as computed by TSC (Fig. 7a). Namely, extrapolating data shown in Fig. 5, we obtain the stability threshold (for $n=1$ interchange mode) at $p_{s} \approx 0.35$, whereas from Fig. 7a we find the stability condition: $p_{s} \geq 0.33$.

The stability diagram for a different pressure profile is shown in Fig. 7b, where

$$
p(x)=0.5 p_{0}\left[\tanh \left(a x+b+c x^{3}\right)+1\right], \quad x=\psi / \psi_{0},
$$

and $a=1.5, b=-3.3$, and $c \geq 0$. Figure $7 \mathrm{~b}$ shows the values of $p_{s}$ corresponding to the marginal stability plotted versus parameter $c$. It is seen that the equilibrium with pressure profile given by Eq. (4) can be stable with respect to interchange modes $(n \geq 1)$ for very small pressure on the separatrix $\left(p_{s} \geq 0.035\right)$. This result has been verified in the initial value linearized MHD simulations, performed for the same pressure profile Eq. (4) and $c=0.2$. In these simulations the $n=1$ interchange mode has been found to be stable for $p_{s}=0.035$ (as compared to $p_{s}=0.04$ from Fig. $7 \mathrm{~b}$ ). Stable equilibrium pressure and current profiles from these simulations are shown in Fig. 8, where pressure is normalized to the external magnetic field pressure $\left(B_{0}^{2} / 4 \pi\right)$, and current is normalized to $e_{i} n_{0} V_{A}$.

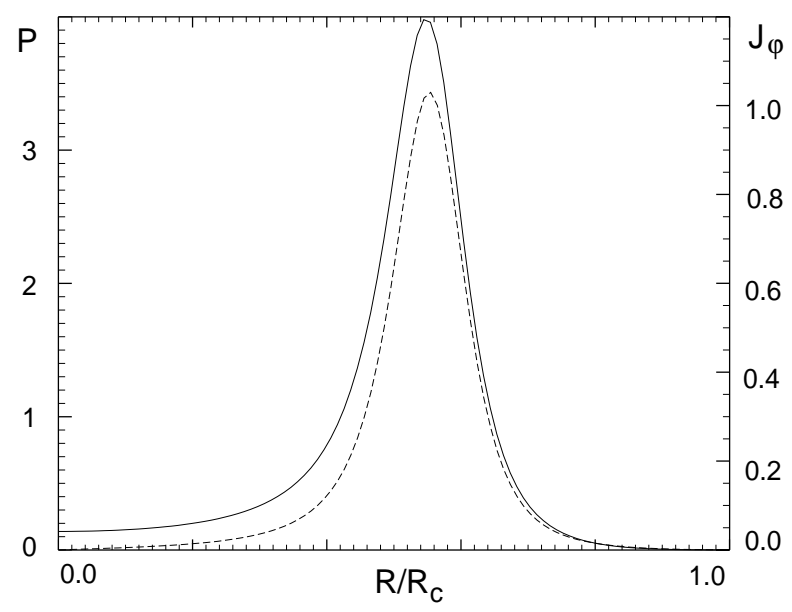

Fig.8 Radial pressure (solid line) and current (dashed line) profiles (at the midplane) stable with respect to the $n=1$ interchange mode. Equilibrium $p(\psi)$ is given by Eq. (4) with $a=1.5, b=-3.3$, and $c=0.2$. 
In summary, we have shown that the $n=1$ interchange mode can be stabilized by increasing the plasma pressure on the open field lines. The stabilization is caused by plasma compression. In general, a relatively large ratio of the separatrix pressure to the maximum pressure is required for stability. However, this value is profile dependent, and equilibrium profiles stable with respect to interchange modes, can be found with much reduced pressure on the separatrix.

\section{NONLINEAR EFFECTS AND $n>1$ MODES}

\section{A. Nonlinear results}

MHD simulations have also been performed to study the nonlinear evolution of the unstable modes, in particular, the $n=1$ external tilt mode and the low $n(n=2-8)$ modes. Our simulations show that nonlinear effects do not stabilize the tilt mode, and a disruption due to growth of the $n=1$, together with $n>1$ modes, has always been observed at the end of the simulation runs. Fig. 9 shows the typical nonlinear evolution of the unstable modes in the oblate FRC with $E=0.69$. Shown are the plasma pressure isosurfaces at $t=0$, $t=2.4 t_{A}$, and $t=2.8 t_{A}$.

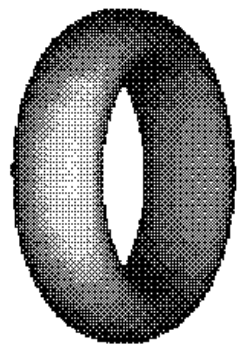

$t=0$

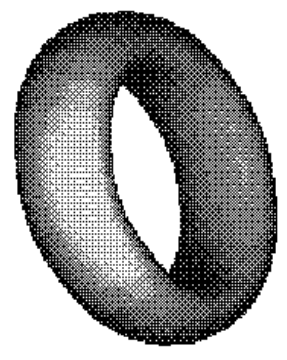

$\mathrm{t}=2.4$

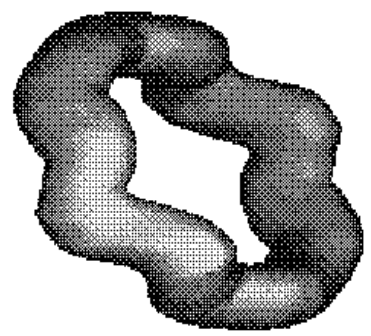

$\mathrm{t}=2.8$

Fig.9 Pressure isosurfaces at $t=0, t=2.4 t_{A}$, and $t=2.8 t_{A}$, from the nonlinear $M H D$ simulation with $E=0.69$ and $n=1$ tilt-like initial perturbation.

Note that these simulations have been performed starting from an $n=1$ tilt-like initial perturbation, and the amplitude of this mode has remained larger than that of the $n>1$ modes until the nonlinear phase. However, higher $n$ modes are generally more unstable 
than the $n=1$ modes, with growth rates several times larger than that of the tilt mode, depending on the profiles and the elongation. For this particular run, the amplitudes of the $n>1$ modes at the end of the simulation are comparable to that of the $n=1$ tilt mode. This can also be seen from Fig. 10, where the time dependences of the kinetic energy of the different $n$ modes are shown. Here, time is normalized to $t_{A}=R_{c} / V_{A}$, which is a characteristic Alfvén time, and $R_{c}$ is the cylinder radius. The calculations shown in Figs. 9 and 10 have been performed for the plasma pressure profile given by Eq. (1), with the mirror ratio $r_{m}=1.3$. For this equilibrium, the most unstable $n>1$ modes are co-interchange modes with the character of an $m=1$ kink mode ( $m$ is a poloidal mode number), and have the displacement vector mostly in the axial direction (Fig. 9).

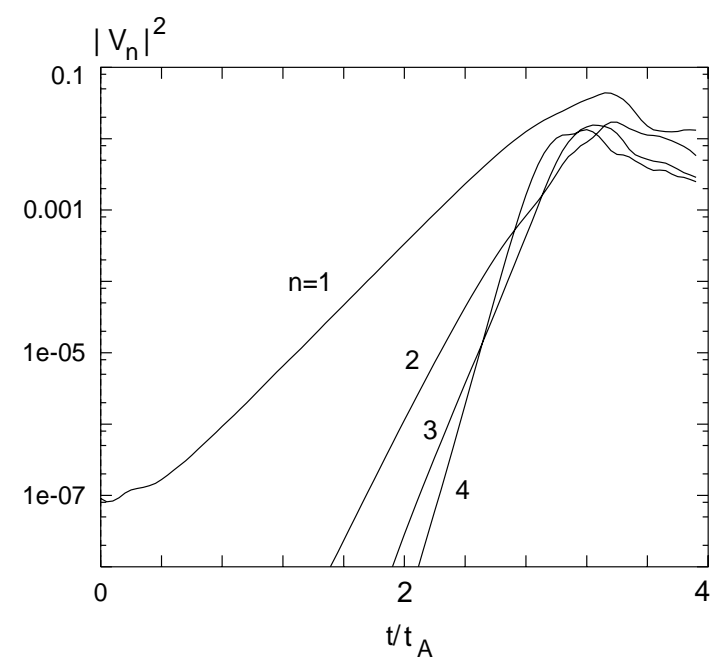

Fig.10 Temporal evolution of the $n=1-4$ kinetic energy from the same calculation as Fig. 9 .

A nonlinear simulation with the same equilibrium profile, Eq. (1), but with a smaller elongation $(E=0.42)$, has also terminated in a disruption due to the growth of the $n>1$ modes. In this case the tilt mode is linearly stable, and the $n=1$ radial shift mode has the growth rate $\gamma=0.8 \gamma_{0}$.

In the nonlinear simulations with a different pressure profile, given by Eq. (2), and with a close-fitting conducting wall used to stabilize the tilt mode, a similar nonlinear behavior has been observed. For the conducting wall position $d=0.06$ (marginally stable tilt mode), we have found the growth rates of the $n=2,3$, and 4 modes to be $\gamma_{2}=1.55 \gamma_{0}, \gamma_{3}=2.61 \gamma_{0}$, 
and $\gamma_{4}=2.63 \gamma_{0}$. The linear structure of these modes corresponds to either that of the interchange $(n=2$ and 4$)$ or co-interchange $(n=3)$ modes. The configuration is destroyed at $t \approx 4 t_{A}$, primarily due to growth of the co-interchange $(n=3$ and 5$)$ perturbations.

\section{B. $n>1$ stability}

Our MHD simulations have shown that in oblate FRCs, the $n>1$ co-interchange modes are strongly unstable, and do not saturate nonlinearly. For the equilibrium with pressure profile given by Eq.(4), the calculated growth rates of the first $n \leq 8$ most unstable modes are shown in Fig. 11. It is seen that at small $n$, the growth rate increases rapidly with $n$, but seems to approach a finite limit $\gamma \sim 7 \gamma_{0}$ at $n \gg 1$. For this equilibrium, the interchange stability condition is satisfied, and the $n \geq 1$ interchange modes indeed appear to be stable. The unstable modes have a $(m=1)$ structure of the co-interchange modes with the displacement vector mostly in the axial direction. The poloidal structure of these modes is similar to that of the $n=1$ tilt mode shown in Fig. 1a, except that for larger $n$ the perturbation becomes localized near the magnetic null. The radial extent of the mode decreases as $n$ increases. For toroidal mode numbers $n \geq 3$, the perturbation amplitude near the separatrix is very small, therefore these modes are essentially internal to the separatrix.

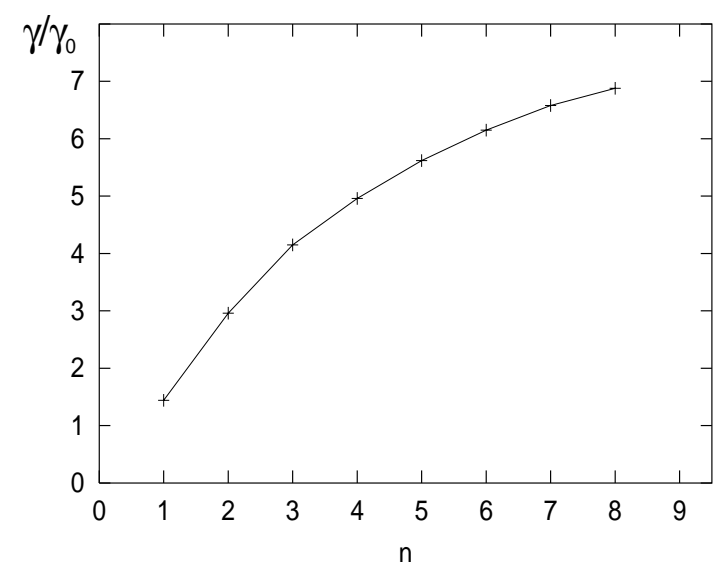

Fig.11 Growth rates of the different $n$ co-interchange modes obtained in simulations with pressure profile given by Eq. (4) with $a=1.5, b=-3.3$, and $c=0.2$. 
The internal nature of $n>1$ modes makes the conducting wall stabilization ineffective. In particular, for the equilibrium given by Eq.(4), the $n=1$ tilt mode is found to be stable for a separation between the separatrix and conducting wall equal to $d=0.09$. For the same separation, the growth rates of the $n=2$ and $n=3$ co-interchange modes are reduced only by $11 \%$ and $6 \%$ respectively, and the growth rate of the $n=8$ mode is very close to that without the conducting wall. For equilibria with smaller $E$, unstable $n>1$ modes also have a $(m=1)$ structure, but in this case the radial polarity mode is more unstable than the axial mode.

It has been shown previously that sheared toroidal rotation has a stabilizing effect on the $n=1$ tilt mode in the prolate $\mathrm{FRC}^{4,7}$, when the rotation rate is sufficiently large. Thus, we have performed MHD simulations to study the effect of sheared flows on the stability of the oblate FRCs, in particular on the $n>1$ co-interchange modes. The simulations have been performed for the pressure profile given by Eq. (1) and angular velocity profile similar to that used by Belova et al. ${ }^{7}$. For this velocity profile, the toroidal rotation frequency, $\omega$, is zero at the magnetic null and at the wall, and has maximum near the separatrix, such that $\Delta \omega=1.44 \gamma_{0}$ for Mach number at the separatrix $M_{s}=V_{s} / V_{A}=2.4$.

In these simulations the growth rate of the tilt mode was reduced from $\gamma=1.2 \gamma_{0}$ $\left(M_{s}=0.0\right)$ to $\gamma=0.78 \gamma_{0}$ for $M_{s}=2.4$. This reduction is considerably smaller than that for the prolate FRC with the same rotation rate. This is because for the same separatrix radius, the growth rate of the tilt mode in the oblate $\mathrm{FRC}(E<1)$ is larger than that in the prolate $(E>1) \mathrm{FRC}$, and for significant reduction in the growth rate to occur, the change in the angular rotation frequency has to be comparable to the characteristic growth rate of the mode $\Delta \omega \gtrsim \gamma_{0}=V_{A} / Z_{s}$. For the prolate $\operatorname{FRC}(E>1)$, a rotation with $M_{s} \gtrsim 1$ is typically required for stability ${ }^{4,7}$. Therefore even larger rotation rates are needed to reduce the growth rate of the tilt mode in the oblate FRC.

The growth rates of the $n>1$ co-interchange modes were reduced due to the sheared rotation, but the reduction was also relatively small. In the simulations with Mach numbers equal to $M_{s}=0.0,1.8$, and 2.4 the growth rate of the $n=4$ mode, for example, has been found to be $\gamma / \gamma_{0}=3.44,3.14$, and 2.12 respectively. For large rotation rates, the radial 
extent of this mode is substantially reduced. The mode becomes more localized near the field null, where the velocity shear is small, thus defeating the stabilizing effect of the sheared rotation. Our simulations, therefore, indicate that the $n>1$ co-interchange modes cannot be stabilized by sheared rotation, unless the rotation rate is extremely large. The rotational stabilization is ineffective both due to large growth rates of the unstable modes, and due to the mode localization near the magnetic null, where the velocity shear is always small.

\section{KINETIC EFFECTS}

The stability of high- $n$ MHD modes in field-reversed geometry has been studied previously using the energy principle approach ${ }^{23-25}$. In the limit $n \gg 1$, it was shown that interchange modes can be stabilized by plasma compressibility for favorable pressure profiles. The radial and axial co-interchange modes are found to be always unstable in the MHD description, with the most unstable flux surfaces being those near the field null ${ }^{23}$. Since the ion Larmor radius is also largest near the field null, these modes $(n \gg 1)$ are likely to be stabilized by kinetic effects ${ }^{24,25}$.

Thus, the intermediate and the low $n>1$ internal kink (co-interchange) modes appear to be the most dangerous in the oblate configuration. We have found that the $n>1$ modes cannot be stabilized by conducting walls, nonlinear effects or sheared rotation. Therefore we consider the possibility of kinetic stabilization for these modes. We have performed nonlinear hybrid simulations for $\bar{s}=4$ and $\bar{s}=2$ to study the bulk ion kinetic effects on the stability of the low $n$ co-interchange modes. The hybrid model and the code used in this study have been described in the previous publication ${ }^{7}$. This model assumes quasineutrality $\left(n_{e}=n_{i}\right)$, cold fluid electrons $\left(p_{e}=0\right)$ and particle ions. The $\delta f \operatorname{method}^{30}$ is used to reduce numerical noise in the simulations. The equilibrium ion distribution function is taken to be $F_{0}=n_{0}\left(m_{i} / 2 \pi T_{i}\right)^{3 / 2} \exp \left(-\varepsilon / T_{i}\right)$, where $\varepsilon=m_{i} v^{2} / 2+e \varphi$ is the particle energy, $T_{i}$ is the uniform ion temperature, and $\varphi$ is the equilibrium electrostatic potential. For this distribution function, $\mathbf{J}_{i}=0$ and all current is carried by the electrons. The equilibrium electric field is equal to $\mathbf{E}=\mathbf{J} \times \mathbf{B} /\left(e n_{e}\right)=T_{i} \nabla n_{i} /\left(e n_{e}\right)$. Since the ion pressure is a scalar for this distribution function, the Grad-Shafranov equation has been solved to find an equilibrium solution. 


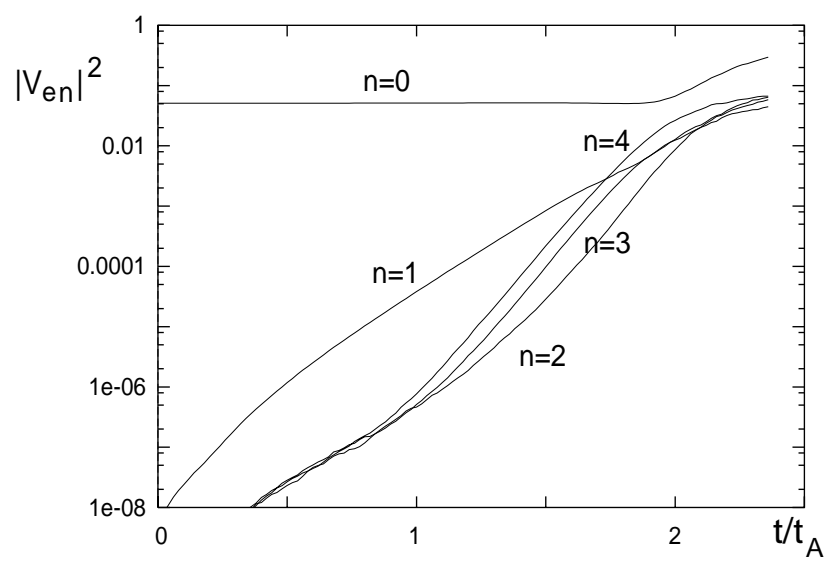

Fig.12 Temporal evolution of the kinetic energy $\left(\left|V_{e}^{2}\right|\right)$ of the $n=0-4$ modes from hybrid simulation with $\bar{s}=4$ and $E=0.69$.

The initial equilibrium was similar to that used in the nonlinear MHD simulations shown in Figs. 9 and 10, with the ion pressure profile given by Eq. (1), $r_{m}=1.3$, and $E=0.69$. The energy plots for $n \leq 4$ modes from nonlinear hybrid simulation with $\bar{s}=4$ are shown in Fig. 12. The initial perturbation for this run was the $n=1$ tilt-like perturbation. Linear growth rates and the nonlinear evolution in this hybrid run are very similar to that in the MHD run, shown in Fig. 10. The calculated growth rates normalized to the MHD growth rates are: $\gamma / \gamma_{m h d}=1.08$ for the $n=1$ mode, and $\gamma / \gamma_{m h d}=0.9$ for the $n=4$ mode $(\bar{s}=4)$. As seen from Fig. 12, in the hybrid simulation the $n>1$ modes grow earlier to larger amplitudes than in the MHD simulation due to relatively high initial numerical noise level in the particle simulation. The configuration is destroyed at $t \approx 2.2 t_{A}$, and the $n=4 \mathrm{kink}$ mode has the largest amplitude in the nonlinear phase. Note that large $n(n \gg 1)$ modes are not well resolved in our simulations, where the cylindrical grid $(r, \phi, z)$ of $(60 \times 32 \times 80)$ grid points is used to represent fields, and $51 \times 51 \times 80$ Cartesian grid is used for particle pushing.

Three typical ion orbits from this nonlinear simulation run are shown in Fig. 13. Figure 13a shows the midplane $(z=0)$ projection of the particle trajectories, and the $r-z$ plane projections are shown in Fig. 13b. The ion trajectories remain confined inside the 
separatrix until $t \approx 2 t_{A}$, when the unstable modes grow to large amplitudes and cause the particles to be lost along the axis.

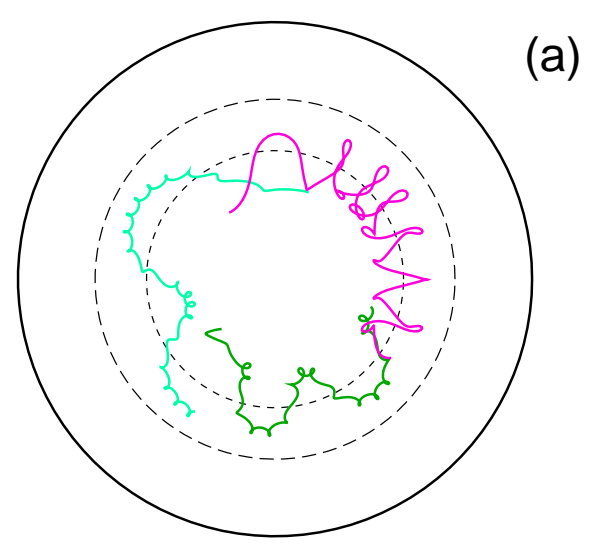

(a)

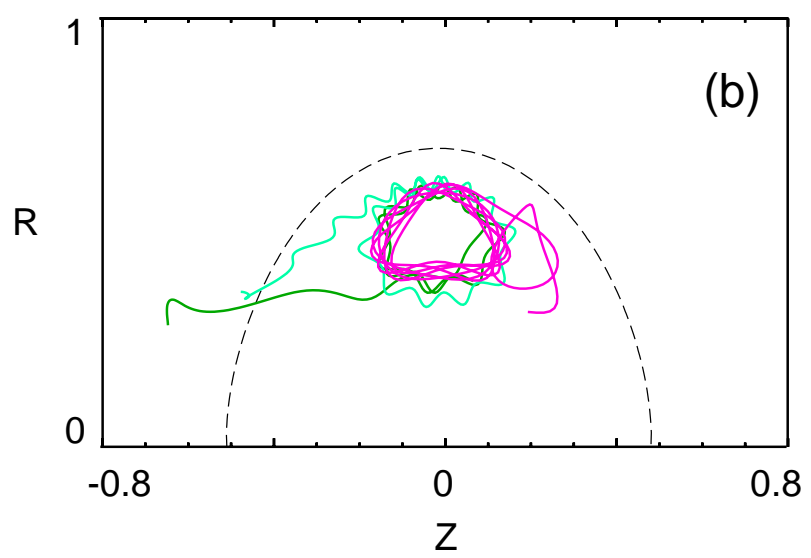

Fig.13 Three ion trajectories from the same nonlinear hybrid simulation as Fig. 12. Shown are (a) projections on the midplane $(z=0)$, and (b) projections on the $r-z$ plane. Dashed lines show the separatrix position, and dotted line show the magnetic null position.

In the simulations with smaller value of $\bar{s}(\bar{s}=2)$, similar results have been obtained. In this case, the normalized growth rates of the $n=1$ and $n=4$ modes are $\gamma / \gamma_{m h d}=1.08$ and $\gamma / \gamma_{m h d}=0.76$ respectively. No nonlinear stabilization has been found in this simulation, and the unstable modes grow to large amplitudes and destroy the configuration at $t \approx 2 t_{A}$.

Our simulations show that both the linear and the nonlinear behavior of low $n \leq 4$ unstable modes is not modified significantly by the finite Larmor radius (FLR) effects for the values of the kinetic parameter $\bar{s} \gtrsim 2$. For the $n=1$ tilt mode this is an expected result, because an approximate condition for FLR effects to be important, $\bar{s} / E \lesssim 0.2-0.5$ (or, equivalently, $\left.\gamma \lesssim \omega^{*}\right)$, implies that $\bar{s}<1$ is needed for the reduction of the tilt mode growth rate in the oblate $\mathrm{FRC}^{3}$. In our simulations, however, the reductions in the growth rates of the $n>1$ modes are also small, even though the diamagnetic frequency $\omega^{*} \sim k_{\phi} \sim n$ is larger. This is because the MHD growth rate increases almost linearly with $n$ for low $n$ modes (Fig. 11). Thus we find that the ion FLR effects alone do not stabilize the low $n$ co-interchange modes, and much smaller values of $\bar{s}(\lesssim 1)$ and, possibly, a non-Maxwellian 
ion distribution function are required to obtain the kinetic stabilization of these modes. Note that our results suggest that the tilt mode suppression found in the oblate FRC experiments ${ }^{15}$ cannot be explained by the FLR effects, since the value of $\bar{s}$ in these experiments was quite large.

The kinetic effects on the stability of the $n=1$ tilt and low $n$ kink (co-interchange) modes have been studied previously for field-reversed ion rings and an ion ring confined plasma $^{26^{-29}}$. When the confined plasma has zero current and pressure, the stability criterion has been shown to be $\mathrm{e}^{26,2}$

$$
(\omega-n \Omega)^{2}>\omega_{\beta}^{2}
$$

where $\omega$ is the mode frequency, $\Omega$ is the toroidal rotation frequency of the beam ions, and $\omega_{\beta}$ is the betatron frequency in the self-field. If this condition is satisfied, the $n=1$ tilt and low $n$ kink modes are MHD stable. With the approximation $\omega \ll \Omega$, the stability condition becomes $n^{2} \Omega^{2}>\omega_{\beta}^{2}$, which can be more easily satisfied for larger toroidal mode numbers. The derivation of this condition assumes a mono-energetic ion beam and thus it is not directly applicable to an equilibrium with thermal ions. It has been shown that if the inequality Eq. (5) holds in an average sense, the kink modes will be MHD stable, but then the resonant instabilities become possible due to the thermal spread in the ion velocities $^{28}$. Detailed numerical calculations are required to self-consistently determine the stability condition in a configuration with a finite spread in the beam ion velocities and a finite confined plasma current.

For the ion distribution function used in the hybrid simulations described above, for majority of particles the betatron frequency, $\left|\omega_{\beta}\right|$, is found to be several times larger than the toroidal frequency, $|\Omega|$, and condition (5) is not satisfied. Thus this equilibrium is MHD unstable, and FLR effects, while reducing the growth rates, do not stabilize the unstable modes completely. A strongly anisotropic ion distribution is therefore required in order to satisfy the inequality (5), and obtain an MHD stable equilibrium in the oblate FRC. A hybrid system, in which field reversal is created both by plasma currents as in the FRC and a low density energetic component of large orbit ions, may provide low $n$ stability, and will be investigated in future work. 


\section{CONCLUSIONS}

Global stability of the oblate FRC has been investigated numerically using linear and nonlinear MHD, and hybrid simulations. The effects of conducting shells, shaping, pressure profile, rotation, and kinetic effects on the stability of $n=1$ and low $n$ MHD modes have been studied. Our simulations show that configurations can be found such that all $n=1$ modes (interchange, external tilt, and radial shift modes) can be stabilized in the MHD regime.

It is shown, that the $n=1$ internal tilt mode, which is considered to be the most dangerous MHD mode in prolate configurations, becomes an external mode when $E<1$, and therefore can be effectively stabilized by close-fitting conducting walls, even in the small Larmor radii (MHD) regime. The marginal separation between the magnetic separatrix and the conducting wall at which the tilt mode becomes stable, depends somewhat on the resistivity profile outside the separatrix and on the current profile near the separatrix. For the equilibria considered, the marginal separation is found to be between $5 \%$ to $10 \%$ of the separatrix radius (at the midplane). The insertion of a central conductor has also been shown to reduce the growth rate of the tilt mode due to line tying effects.

The stability of the oblate FRC to tilt and shift modes, in general, is found to be very similar to that of spheromak plasmas ${ }^{14,16,17}$. In particular, the tilt mode stability improves with oblateness, and, at small elongations, $E \leq 0.55$, (i.e. larger mirror ratio of the external coils) the radial shift mode becomes more unstable than the tilt mode.

The $n=1$ interchange mode stability is strongly pressure profile dependent, with the growth rate being smaller or comparable to that of the tilt mode. An addition of a uniform background pressure reduces the growth rate and the mode becomes more localized near the magnetic null. An equilibrium with a relatively peaked pressure profile has been found which is stable with respect to the $n \geq 1$ interchange modes for a small plasma pressure at the separatrix (3.5\% of the maximum pressure). Our simulations therefore show that all $n=1$ modes can be stabilized in the MHD regime, provided that the pressure profile is sufficiently peaked (interchange stable), the elongation is in the range $1>E \gtrsim 0.6$, and a conducting wall is used to stabilize the tilt mode. 
However, in the nonlinear simulations the growth of MHD modes with low to intermediate $n$ has been observed. We have shown that these modes have large linear growth rates, which increase with $n$, and do not saturate nonlinearly. Thus, even in the cases when $n=1$ modes are stabilized, the oblate FRC still disrupts due to the growth of the $n \sim 3-5$ perturbations.

For an equilibrium stable to interchange modes, the $n>1$ modes have a structure of the co-interchange $(m=1 \mathrm{kink})$ modes with a displacement vector being in the radial or axial direction. Depending on the separatrix elongation, the most unstable modes are axial (larger $E)$ or radial $(E \lesssim 0.4)$ kink modes. As the toroidal number increases, the radial extent of these modes decreases, and the modes become more localized near the magnetic null. The localization of the unstable modes near the field null makes the conducting wall and the rotational stabilizations ineffective, but suggests that kinetic effects can be important.

Our hybrid simulations with $\bar{s}=2$ and $\bar{s}=4$ confirm that for an oblate FRC, the FLR effects on the $n=1$ tilt mode linear stability are small due to the large MHD growth rate of this mode ${ }^{3}$ (for $E<1$ ). The stabilizing effect of the FLR on the low $n>1$ co-interchange modes is also found to be small, and the nonlinear evolution of the unstable modes in the hybrid runs has been shown to be very similar to that in the MHD simulations. Thus the thermal ion FLR effects (for $\bar{s} \geq 2$ ) are not sufficient to completely stabilize the $n>1$ modes. However, the possibility still exists that these mode can be stabilized by energetic ion beams, which was not considered in this paper.

In previous work ${ }^{24,25}$, it has been shown that an energetic ion beam carrying a large fraction of the total current (more than a half), can stabilize the $n \gg 1$ co-interchange modes. This stabilization is related to the fact that for large $n$, the energetic ions do not interact with the wave, but effectively reduce the fraction of the current carried by the background plasma (destabilizing term). Since the magnetic tension (stabilizing term) remains the same, the mode becomes stable when the fraction of the current carried by the beam is sufficiently large.

For the low $n>1$ co-interchange modes, the energetic ion beam contribution is stabilizing if the condition Eq. (5) is satisfied ${ }^{17,26,27}$. In this case the stabilization is caused by the 
resonant wave-beam interaction, and the inequality Eq. (5) is required for the beam particles to respond out of phase (stabilize) with the wave. Numerical simulations of the oblate FRC with energetic ion beams or a strongly anisotropic ion distribution function are required to establish the kinetic stabilization of low $n$ modes, in particular to find the condition (fraction of beam current) necessary for stability of the hybrid beam/thermal plasma system.

In summary, we have shown that all $n=1$ modes (including the tilt mode) can be stabilized in the oblate FRC in the small Larmor radius (MHD) limit. The stability to $n>1$ interchange modes can be achieved for a favorable pressure profile and a finite separatrix beta. However, the stability of the low $n$ co-interchange (internal kink) modes remains an issue. Future work will focus on evaluating the effectiveness of an energetic ion beam component in stabilizing these modes.

\section{ACKNOWLEDGMENTS}

The authors acknowledge many useful discussions with J. Finn, Y. Omelchenko, W. Park, L. Steinhauer, and M. Tuszewski. This work was supported by DOE contract \#DE-AC02-76-CHO-3073. 


\section{REFERENCES}

${ }^{1}$ M. Tuszewski, Nucl. Fusion 28, 2033 (1988).

${ }^{2}$ J.M. Finn and R. N. Sudan, Nucl. Fusion 22, 1443 (1982).

${ }^{3}$ A. Ishida, H. Momota and L. C. Steinhauer, Phys. Fluids, 31, 3024 (1988).

${ }^{4}$ R. D. Milroy, D. C. Barnes, R. C. Bishop, R. B. Webster, Phys. Fluids B 1, 1225 (1989).

${ }^{5}$ D. C. Barnes, J. L. Schwarzmeier, H. R. Lewis, C. E. Seyler, Phys. Fluids 29, 2616 (1986).

${ }^{6}$ D. C. Barnes and R. D. Milroy, Phys. Fluids B 3, 2609 (1991).

${ }^{7}$ E. V. Belova, S. C. Jardin, H. Ji, M. Yamada, and R. M. Kulsrud, Numerical Study of Tilt Stability of Prolate Field-Reversed Configurations, to appear in Phys. Plasmas (2000).

${ }^{8}$ E. V. Belova, S. C. Jardin, H. Ji, R. M. Kulsrud, W. Park, M. Yamada, Global Stability of the Field Reversed Configuration, to appear in Plasma Physics and Controlled Nuclear Fusion Research, (Proc. 18th Int. Conf. Sorrento, 2000), (International Atomic Energy Agency, Vienna, 2000).

${ }^{9}$ R. Horiuchi and T. Sato, Phys. Fluids B 1, 581 (1989).

${ }^{10}$ H. Ji, M. Yamada, R. Kulsrud, N. Pomphrey, and H. Himura, Phys. Plasmas 5, 3685 (1998).

${ }^{11}$ E. V. Belova, S. C. Jardin, H. Ji, M. Yamada, and R. M. Kulsrud, Bull. Am. Phys. Soc. 44, 85, (1999).

${ }^{12}$ M. N. Rosenbluth, M. N. Bussac, Nucl. Fusion 19, 489 (1979).

13 S. C. Jardin and U. Christensen, Nucl. Fusion 21, 1665 (1981).

${ }^{14}$ M. Yamada, R. Ellis, Jr., H. P. Furth et al., in Plasma Physics and Controlled Nuclear Fusion Research, (International Atomic Energy Agency, Vienna, 1983), Vol. II, p. 265.

${ }^{15}$ Y. Ono, Bull. Am. Phys. Soc. 44, 85 (1999); T. Matsuyama, L. Y. Wah, Y. Ono, M. Katsurai, 
Bull. Am. Phys. Soc. 44, 44 (1999).

16 S. C. Jardin, M. S. Chance, R. L. Dewar, R. C. Grimm, D. A. Monticello, Nucl. Fusion 21, 1203, (1981).

17 J.M. Finn and A. Reiman, Phys. Fluids 25, 116 (1982).

18 J.M. Finn, Phys. Fluids 27, 2973 (1984).

${ }^{19}$ D. D. Ryutov, presented at International Sherwood Fusion Theory Meeting, March 2000, Los Angeles, CA (2000).

${ }^{20}$ L. Sparks, J. M. Finn, and R. N. Sudan, Phys. Fluids 23, 611 (1980).

${ }^{21}$ I. B. Bernstein, E. A. Frieman, M. D. Kruskal and R. M. Kulsrud, Proceedings of the Royal Society, 244, 17 (1958).

${ }^{22}$ S. C. Jardin, N. Pomphrey and J. L. Delucia, J. Comput. Phys., 66, 481 (1986).

${ }^{23}$ P. L. Pritchett, Phys. Fluids 24, 864 (1981).

${ }^{24}$ J. M. Finn, Phys. Fluids 24, 274 (1981).

${ }^{25}$ L. Sparks and R. N. Sudan, Phys. Fluids 27, 626 (1984).

${ }^{26}$ R. V. Lovelace, Phys. Fluids 19, 723 (1976).

${ }^{27}$ C. B. Ruchti and R. V. Lovelace, Phys. Fluids 27, 1789 (1984).

${ }^{28}$ J.M. Finn and R. N. Sudan, Phys. Fluids 22, 1148 (1979).

${ }^{29}$ A. Friedman, R. N. Sudan and J. Denavit, Phys. Fluids 29, 3317 (1986).

${ }^{30}$ S. E. Parker and W. W. Lee, Phys. Fluids B 5, 77 (1993). 
The Princeton Plasma Physics Laboratory is operated by Princeton University under contract with the U.S. Department of Energy.

\author{
Information Services \\ Princeton Plasma Physics Laboratory \\ P.O. Box 451 \\ Princeton, NJ 08543
}

Phone: 609-243-2750

Fax: 609-243-2751

e-mail: pppl_info@pppl.gov

Internet Address: http://www.pppl.gov 